\title{
Éditorial \\ Contextes et transmissions : les apports de la recherche en éducation. Les exemples de la Polynésie française, de la Nouvelle Calédonie et de l'Océanie
}

\author{
Rodica AILINCAI ${ }^{1}$ et Antoine DELCROIX ${ }^{2}$ \\ ${ }^{1}$ Université de la Polynésie française - EASTCO (EA 4241) \\ ${ }^{2}$ Université des Antilles - CRREF (EA 4538)
}

Ce numéro de la revue Contextes et Didactiques trouve son origine dans les Journées de la Recherche en Éducation (JRE 2018), qui ont eu lieu à l'université de la Polynésie française en 2018 ${ }^{1}$. L'ambition de cette manifestation était de créer des ponts entre la recherche, la formation (des enseignants) et le terrain, ce triptyque favorisant la structuration d'un espace de recherche fondamentale en éducation basé sur les besoins de l'enseignement, de l'éducation et de la formation. Initié au sein de l'université de la Polynésie française par l'Équipe d'Accueil Sociétés traditionnelles et contemporaines en Océanie (EASTCO) et l'ESPE de la Polynésie, le colloque a rassemblé plusieurs partenaires : le Vice-rectorat de Polynésie française, la Direction Générale de l'Éducation et des Enseignements de la Polynésie française et le Centre de recherches et de ressources en éducation et formation (CRREF), équipe d'accueil de l'université des Antilles.

Dans un contexte polynésien où la recherche en éducation doit composer avec la double contrainte d'isolement et d'éloignement, ce colloque a permis de créer des liens avec des grands centres nationaux et internationaux de production de connaissances. À un niveau plus régional, il a contribué à renforcer les liens entre formation initiale et continue, en permettant d'envisager une formation des enseignants appuyée sur la recherche en éducation en général et plus particulièrement celle qui s'intéresse aux relations entre contextes et éducation. La recherche en éducation en Océanie, s'inscrivant dans un contexte post-colonial (Molinié, 2016), doit être au service des populations locales et promouvoir une recherche collaborative avec les acteurs de terrain (Salaün et Vernaudon, 2014). Toutefois, cette exigence que les chercheurs et partenaires locaux se donnent, peut se répercuter sur la communication des résultats de la recherche, parfois au risque de l'autocensure : par leur insertion dans le tissu social et professionnel polynésien, les chercheurs sont sensibles aux effets que pourraient produire leurs écrits (Fillol et Le Meur, 2014). C'est pourquoi, les liens avec des laboratoires externes, l'initiation de recherches et de manifestations scientifiques permettent d'inscrire les problématiques locales dans de grands enjeux théoriques plus universaux. Les JRE 2018 ont réuni 63 communicants venant du monde de la recherche (22), de l'enseignement universitaire (15), de la formation (14 étudiants de niveau master et 7 doctorants), du terrain (5), autour de 37 communications et deux tables rondes.

\footnotetext{
${ }^{1}$ Voir : http://espe.upf.pf/jre/.
} 
Devant la réponse forte des différents acteurs du monde éducatif et de la recherche à l'appel à contribution des JRE 2018, les acteurs de cette manifestation et le comité de rédaction de Contextes et Didactiques ont décidé de consacrer un numéro thématique de la revue aux questions posées lors de cette manifestation, notamment autour de la liaison entre les résultats des recherches théoriques et leurs déclinaisons dans les pratiques pédagogiques et les actions de formation initiale et continue en contexte. Finalement, l'appel à contribution correspondant a rencontré un succès certain en élargissant les points de vue exposés lors des JRE 2018. Les contributions retenues et composant ce numéro peuvent être regroupées en plusieurs sousensembles que nous présentons maintenant.

Dans le cadre d'un système éducatif restant fortement centralisé, les prescrits sont élaborés en France par le gouvernement central et s'appliquent, en règle générale, dans l'ensemble du territoire, avec quelques possibilités d'ajustement. Ainsi, des adaptations sont possibles concernant l'enseignement des langues et cultures régionales dont l'« enseignement est favorisé prioritairement dans les régions où elles sont en usage $»^{2}$. De plus, dans certaines des collectivités d'Outre-mer (Guadeloupe, Guyane, Martinique, La Réunion, Mayotte), des adaptations sont également possibles dans des champs disciplinaires précis, comme l'histoire et la géographie, les sciences de la vie et de la terre. Enfin, la Nouvelle Calédonie et la Polynésie française jouissent d'une plus grande autonomie dans la détermination des programmes scolaires. Néanmoins, dans ces territoires, l'organisation globale du système demeure assez similaire à celle en vigueur dans le reste de la France, ce qui fait que l'école y reste grandement l'école du socle. En lien avec ce point, Joël Lebeaume montre, dans une contribution intitulée «L'école du socle: un curriculum. Perspectives pour les recherches didactiques ", comment cette vision de l'école constitue une inflexion importante des politiques éducatives en France. Il inscrit son étude dans une triple perspective sociohistorique, comparative et didactique. Dans la suite de cette étude, Antoine Delcroix questionne le concept de contextualisation, sous le titre "Contextualisation didactique : un concept en tension?», dans les systèmes éducatifs fortement centralisés, en prenant l'exemple du système français : quelle place les phénomènes de contextualisation y prennentils ? Comment les acteurs du système appréhendent-ils cette question.

Ces deux contributions fournissent le soubassement de ce numéro qui montre comment les travaux de recherche éclairent les problématiques de contextualisation dans les territoires de Polynésie française et de Nouvelle Calédonie. Les six contributions, qui forment le cœur du dossier thématique de ce numéro, se déploient selon deux axes.

Le premier axe questionne certains aspects de la relation des parents à l'école, dans le contexte familial polynésien dont les caractéristiques sont très éloignées de celui de l'Hexagone, modèle sur lequel l'institution scolaire définit - au moins de manière implicite les relations entre les systèmes éducatif et familial (Salaün et Le Plain, 2018). Dans une première contribution relative à cet axe, intitulée "L'implication parentale en Polynésie française. Le cas de deux écoles contrastées sur le plan socioéconomique », Émilie Guy et Rodica Ailincai montrent l'influence des paramètres sociaux sur l'implication des parents, en confirmation de résultats déjà observés et dont la littérature rend compte. Mais les auteurs mettent également en exergue des différences dans la relation des parents à l'école par rapport

\footnotetext{
${ }^{2}$ Code de l'éducation, article L310. Disponible en ligne :

https://www.legifrance.gouv.fr/affichCode.do;jsessionid=D77E9728EC1B6D980831823F64045CE2.tplgfr44s_2

?idSectionTA=LEGISCTA000006182403\&cidTexte=LEGITEXT000006071191\&dateTexte $=20130710$.

Consulté le 7 novembre 2019.
} 
à d'autres lieux. Ces différences sont probablement en rapport avec le contexte sociolinguistique de la Polynésie et avec les dynamiques culturelles et identitaires qui s'y déploient. Dans la seconde contribution liée à cet axe, intitulée «Les styles interactifs des parents polynésiens dans le cadre de l'aide aux devoirs : l'exemple de la langue tahitienne ", Ernest Marchal et Rodica Ailincai présentent une étude exploratoire sur les interactions mèreenfant à visée d'apprentissage. Cette étude montre une prédominance d'un style directif chez les mères qui, par ailleurs, s'expriment majoritairement en français pour accompagner leur enfant dans une lecture d'un texte en langue tahitienne. Des éléments explicatifs d'ordre socioculturel sont avancés, qui invitent à une comparaison avec des études similaires réalisées dans d'autres contextes (comme par exemple : Ailincai, Alì et Alby, 2018 ; Jeannot-Fourcaud et Anciaux, 2014 ; Qribi, 2008).

Le second axe s'intéresse plus particulièrement au système éducatif formel, au travers de regards portés sur l'apprentissage des élèves, sur la formation des enseignants et des étudiants de niveau master et sur la mise en œuvre de démarches pédagogiques. Trois contributions questionnent l'apprentissage et l'enseignement des langues. Pour les deux premières, le terrain d'enquête est la Polynésie française et pour la dernière la Nouvelle Calédonie. Sous le titre «Analyse des interactions dans la classe d'anglais à l'école élémentaire : une étude sur l'implication des apprenants ", Zehra Gabillon s'intéresse à l'implication des apprenants dans des leçons d'anglais langue vivante étrangère à l'école primaire, mesurée au travers d'une analyse des interactions en classe. L'étude montre notamment que les choix pédagogiques des enseignants ne favorisent que peu les interactions entre élèves (le temps de parole de l'enseignant domine). En effet, les activités proposées, "très mécanistes », ne permettent pas une réelle implication cognitive des élèves et le développement d'une communication entre eux. Comme élément explicatif de ces constats, on peut formuler l'hypothèse d'une question de formation des enseignants aux démarches pédagogiques encourageant les interactions entre élèves. Cette hypothèse est éclairée par la contribution suivante, sous le titre "Quelle formation au numérique éducatif pour les enseignants de langues vivantes ? Retour sur le projet 'Ia Ora Numérikids », où Guilène Révauger s'intéresse à la question de la formation au numérique éducatif des futurs professeurs du secondaire de langues vivantes en Polynésie française : le virage vers le numérique s'y opère rapidement, mais un écart demeure avec l'ensemble français dans l'équipement des établissements scolaires. Une expérience menée sur des activités en anglais et en tahitien à destination d'élèves de collège sert de base à l'auteure, qui montre, dans une perspective actionnelle, les bénéfices du travail collectif chez les élèves et de l'engagement des professeurs dans des démarches collaboratives. Dans une contribution intitulée «La créativité en formation professionnalisante: un processus émancipateur », Véronique Fillol, Elatiana Razafimandimbimanana et Stéphanie GeneixRabault décrivent et analysent une formation professionnelle, le Diplôme Universitaire en Didactique du français langue étrangère/seconde, spécialité « Interculturalités » mis en place à l'université de la Nouvelle-Calédonie. Cette formation rompt avec des pratiques de contextualisation faibles, en plaçant le déjà-là des apprenants au cœur des processus d'apprentissage. L'expression de ce déjà-là - le plus souvent pluriculturel et plurilingue - est sollicité au travers de productions dépassant les formes universitaires classiques (par exemple : des récits plurilingues, interculturels et plurisensoriels) développant ainsi, à des fins de professionnalisation, des qualités de créativité. 
Cet axe se clôt par une contribution de Sabine Dufay, intitulée "Représentations et mise en œuvre de la pédagogie d'investigation à l'école élémentaire : études de cas », qui part du constat que les programmes de 2016 adaptés à la Polynésie Française pour les cycles 2 et $3^{3}$ s'inscrivent dans une approche socioconstructiviste identique à celle des programmes en vigueur dans le reste du territoire français (à l'exception de la Nouvelle-Calédonie). Dans une recherche à caractère exploratoire, l'auteure compare les représentations et les pratiques d'une professeure des écoles stagiaire et d'une professeure des écoles expérimentée en matière de pédagogie d'investigation. Cette étude contribue à la mise au point d'une méthodologie qui permet de mesurer la place accordée à la pédagogie d'investigation par les professeurs des écoles en Polynésie, dans une perspective de formation initiale et continue des enseignants.

Ce numéro s'achève par une contribution de François-Xavier Bernard, intitulée «Tutelle et médiation enseignante en situation d'activité instrumentée: le cas d'une séquence de programmation à l'école élémentaire ». Hors dossier principal, ce texte rappelle cependant qu'aux contextes socioculturels et sociolinguistiques largement au cœur de ce numéro, se superposent d'autres objets de contextes, comme les évolutions technologiques et les instructions officielles les concernant. Il s'agit ici, dans le cadre de l'apprentissage de la programmation à l'école primaire, de rendre compte de l'utilisation par un enseignant de cycle 3, d'un logiciel de programmation dont il n'est pas familier. Le professeur assume, dans le cas de l'étude, une posture de "tuteur naïf " s'appuyant, le cas échéant sur des élèves experts, ce qui contribue à une co-construction des connaissances. La question soulevée ici pourrait être celle de la formation des enseignants aux apprentissages spécifiques au numérique.

Tout comme les contributions précédentes, portant sur l'environnement socioculturel et sociolinguistique, celle-ci met en évidence la manière dont la recherche éclaire les pratiques enseignantes, en relation avec les objets qui les entourent ou les constituent. En écho à l'objectif des JRE 2018, nous espérons que les différentes contributions composant ce numéro pourront servir, même modestement, à la mise en place d'une formation rénovée des enseignants et des personnels d'éducation en particulier dans les Outre-mer, éclairée par des travaux de recherche issus des multiples champs contributifs à la recherche en éducation. C'est, en tout cas, le vœu que nous formulons.

\section{Références bibliographiques}

Ailincai, R., Alì, M. et Alby, S. (2018). Epistemic Activities, Schooling and Parenting Styles: A Case Study in French Guiana Rural Contexts. Contextes et Didactiques, 12, 60-84. Disponible en ligne: https://journals.openedition.org/ced/1119. Consulté le 12 septembre 2019.

Fillol, V. et Le Meur P.-Y. (dir.) (2014). Terrains océaniens : enjeux et méthodes. Paris : L'Harmattan, Collection Cahiers du Pacifique Sud Contemporain.

Jeannot-Fourcaud, B. et Anciaux, F. (2014). Pratiques, représentations et attitudes linguistiques à l'école en Guadeloupe : éléments pour une didactisation de l'alternance codique. Dans B. Jeannot-Fourcaud, A. Delcroix et M.-P. Poggi (dir.), Contextes, effets de contextes et didactiques des langues (p. 67-94). Paris: L'Harmattan, Collection Logiques sociales.

\footnotetext{
3 Disponible en ligne: http://www.education.pf/wp-content/uploads/2016/08/2016-Programmes_Cycles-23Version_29-07-2016.pdf. Consulté le 7 novembre 2019.
} 
Molinié, M. (dir.) (2016). Accompagnement sociobiographique en contexte post-colonial : plurilinguisme, émancipation, formation. Contextes et Didactiques, 8. Disponible en ligne : https://journals.openedition.org/ced/296. Consulté le 10 octobre 2019.

Qribi, A. (2008). Éducation familiale, expérience scolaire et orientations identitaires chez les jeunes adultes issus de l'immigration maghrébine en France. Thèse de doctorat non publiée, Université de Toulouse Le Mirail.

Salaün, M. et Le Plain, E. (2018). L'école ambiguë : histoires de familles à Tahiti. Paris : L'Harmattan. 